\title{
Instantaneous, Short-Term and Predictive Long-Term Power Balancing Techniques in Intelligent Distribution Grids
}

\author{
Alexander Suzdalenko ${ }^{1,2}$ and Ilya Galkin ${ }^{1}$ \\ ${ }^{1}$ Riga Technical University, Riga, Latvia \\ aleksandrs.suzdalenko@rtu.1v, gia@eef.rtu.lv \\ ${ }^{2}$ Tallinn University of Technology, Tallinn, Estonia
}

\begin{abstract}
An increased number of distributed small generators connected to the power grid allows higher total efficiency and higher stability of electrical power supply by exporting energy to the grid to be achieved during peak demand hours. On the other hand, it poses new challenges in structuring and developing the control approaches for these distributed energy resources. This paper proposes an improved method of real-time power balancing targeted to reaching long-term energy management objectives. The novel long-term energy management technique is proposed, that is based on load categorization and regulation of energy consumption by regulating electricity price function estimated with the proposed mathematical model. The method was evaluated by a LabVIEW model by simulating various types of loads. The price function for the defined energy generation pattern from renewable energy sources was obtained.
\end{abstract}

Keywords: Intelligent Distribution Grid, Nanogrid, Energy Management, Instantaneous Power Balancing, Short-Term Energy Management, Pricing.

\section{Introduction}

The dynamic of worldwide installed power utilizing renewable energy sources (RES) is increasing year by year [1], showing global awareness of climate change and the footprint of human behavior on the nature: like mining activities that change the landscape and damages caused by oil plants. Another problem is related to the total effectiveness of energy from the primary fuel. It relates to the total system efficiency of energy delivery to the end-user from a mining site, which includes energy losses at middle stages, like use of energy at the mining stage, energy conversion losses, transmission (mechanical and electrical) losses, as well as end-device efficiency. Consequently, locally generated energy (especially from renewable energy sources) is preferable because it excludes most of the mentioned losses.

As more distributed small generators are connected to the power grid, higher total efficiency and higher stability of electrical power supply by exporting energy to the grid during peak demand hours. On the other hand, it poses new challenges in structuring and developing the control approaches for these distributed energy resources. 
Thus, it can be concluded that the centralized generation is moving towards distributed, plug-and-play type of generation that requires a special control approach. Implementation of centralized control for such a dispersed grid is unacceptable, thus some kind of distributed control is to be realized.

The advantages of Multi-Agent System (MAS) use in control applications described in [2] demonstrate the effectiveness of this approach in the organization and management of the intelligent distribution grid in large-scale applications.

This paper discusses different energy management approaches for an intelligent grid that consists of RES, energy storage devices and controllable loads. An improved instantaneous energy management method is described targeted to obtaining longterm objectives and a novel long-term energy management approach is proposed based on load categorization and regulation of energy consumption by regulating electricity price function.

\section{Relationship to Internet of Things}

Concerning growing interest in intelligent systems that acquire data from network sensors, much higher predictive operation of household appliances is expected [3]. The concept named Internet of Things delivers the idea of connecting each smart appliance to the Internet, helping to communicate with the data center and providing advanced functionality (such as predictive connection of loads) that could help to solve the problem of peak demand, by scheduling of distributed generators and shedding the end-user's load or in other words, enabling demand-side management [4] [5].

The idea of Internet of Things covers a large functionality of the smart nodes, but this article uses a simplified model to test the long-term scheduling approach by using a bidirectional communication between the sources and loads on the one side and the data center on the other side.

\section{State of the Art in Energy Management}

Currently a major effort is focused on the approaches that keep energy balance in small-scale networks, such as nanogrids [6][7], microgrids [8]. The energy management approaches are divided into groups which differ by the period of management: instantaneous (real-time) energy management, keeping balance in the period of milliseconds or seconds, short-term energy management, with periods of seconds to hours and long-term energy management, which defines energy management strategy to fulfil objectives for weeks and months.

\subsection{Instantaneous Energy Management}

The operation in islanded mode requires special control techniques for energy generation units as they should regulate the output power with the demand of the local grid. For this reason the droop control approach is mostly utilized both in AC and in DC 
power distribution grids, which defines the method of power sharing between various sources by means of definition of different droops according to their nominal power [9].

The droop control differs between AC and DC grids, because in a DC grid only supply bus voltage should be regulated, but in an AC grid - voltage and frequency. AC droop control is realized in the following way - the amount of active power is tight with the frequency, but reactive power with voltage reference. When a generator's output power becomes more capacitive, it increases the voltage reference, which increases the output power of the generator, at the same time decreasing the output frequency, thus sharing power with other generators.
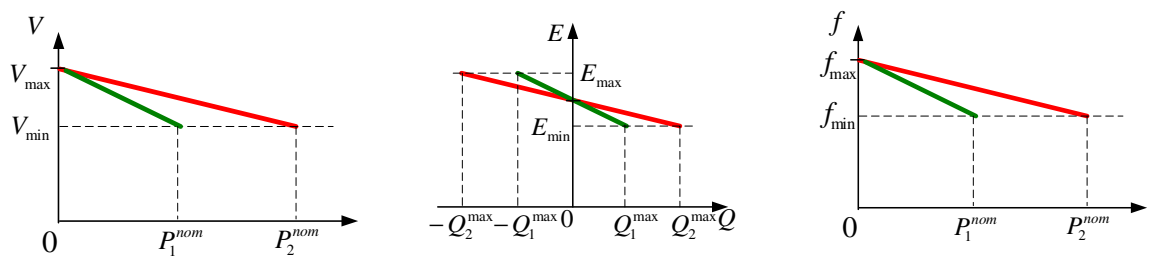

Fig. 1. Droop control method - V-droop for DC grid, Ef-droop for AC grid

From the point of view of energy management, DC distribution grids provide less problems, as only single parameter should be regulated within the defined levels. In contrast, energy management in an $\mathrm{AC}$ grid requires more attention due to inherent problems of reactive power fluctuations, harmonics, unbalances, as well as certain difficulties for generators to operate in no load conditions. These problems are compared in the table below.

Table 1. Comparison of instantaneous power balancing control challenges in AC and DC grids

\begin{tabular}{|l|c|c|}
\hline \multicolumn{1}{|c|}{ Problem } & AC grid & DC grid \\
\hline $\mathrm{P}-$ active power balance & $\bullet$ & $\bullet$ \\
\hline $\mathrm{Q}-$ reactive power balance & $\bullet$ & \\
\hline F - frequency control; synchronization; & $\bullet$ & \\
\hline Harmonics, 3-phase unbalance & $\bullet$ & \\
\hline $\mathrm{R}=\infty$ - no load condition & $\bullet$ & $\bullet$ \\
\hline Imax - Inrush currents & $\bullet$ & \\
\hline
\end{tabular}

\subsection{Short-Term Energy Management}

Instantaneous power balancing provides power sharing approach between various of sources. However it does not provide information about the resources of the grid, for instance, islanded DC grid consisted of $1 \mathrm{~kW}$ source and $500 \mathrm{~W}$ load will operate at the same voltage level as second islanded DC grid, which consists of $10 \mathrm{~kW}$ source and $5 \mathrm{~kW}$ load. It means, that in one case there is no possibility to supply additional 1 $\mathrm{kW}$ load, but in other grid - there is possibility. Thus, special control approach is needed to overcome this issue. 
In [10] the DC bus signalling (DBS) approach is applied to this problem. The control technique is based on different disconnection delays for different priority loads when DC bus undervoltage condition is occurred, thus enabling to lowest priority loads with shorter delays to disconnect faster, releasing power for higher-priority loads. The drawbacks of described approach are the following: the delays are set constantly, meaning that the controllability of such approach is limited; there is always trade-off between the delay periods (with longer periods it is possible to define more steps of load prioritization) and the quality of DC bus voltage, as the longer are delays, the bigger are voltage dips.

In our opinion, the communication hardware should be used to provide better short-term energy management that would eliminate unwanted voltage dips.

\subsection{Long-Term Energy Management}

According to literature [11], with proper control of microgrids it is possible to realize long-term objectives, such as the reduction of $\mathrm{CO} 2$ emissions by a certain percentage or export a defined amount of energy to the grid during some period (week, month or even year), which could be required by contract statements or transmission network operator's tasks.

It is recommended to solve that problem with artificial neural networks [12], which will successfully predict the amount of generated power by RESs. Another solution is to use genetic algorithms [13] that help to choose an optimized operation schedule for energy storage components providing reliable power supply and keeping lifetime of energy storages longer due to full charge and discharge cycles. An additional solution is provided by a pricing approach [14] based on changing the price per electrical energy. Different loads and sources behave according to an internal logic - how to operate under certain price limits. This solution results in additional load when the price is low and vice versa, load shedding when the price is high. However, it is not possible to predict amount of switched on or off load with this solution.

\section{Contribution of Research}

Instantaneous energy management approach based on the droop control method can be used to obtain long-term objectives in energy management by regulating the reference values of DC bus voltage for a bidirectional AC/DC converter that will influence the consumed energy in the local grid.

The proposed method of long-term energy management is based on the mathematical model of price function estimation. It can be interpreted as a graphical approach of predictive energy generation surface intersection with acquired operation schedule of categorized loads, which can also be drawn as a 3D surface, thus enabling the control of the intelligent grid by providing only price function information.

\subsection{Improved Instantaneous Power Balancing}

Our proposal is to use the well known droop control method for keeping power balance in the grid, but with variable reference values of a common point interface (CPI) 
converter. It is especially suitable for DC supply grids that always operate under the droop control method, as these are not connected to the utility grid directly, but through an AC/DC interface converter, which imports or exports energy to or from the grid, depending on the DC bus voltage. We propose to use variable reference value of the DC voltage grid for an interface converter that will influence the operation of local grid elements, for instance by decreasing the DC bus voltage reference value to few per-cents below the nominal value, the unnecessary loads will not operate or will operate at the highest energy saving mode and as a result, more energy will be exported to the grid.

In conclusion, it is possible to fulfil long-term objectives with a CPI converter which regulates its reference value for the regulation of a local grid operation mode, as other grid elements choose their operation mode in accordance with the grid voltage - the parameter available for all elements of the grid.

\subsection{Novel Long-Term Energy Management Approach}

Our proposal is to define categories of household appliances which switch and operate at different conditions:

1. Time-triggered load (see Fig.2a) - pumps, general illumination etc. - the load which basically switches on at a defined time;

2. Price-triggered load (see Fig.2b) - energy storage element, washing machines etc. - the load which turns on when the price is within an acceptable level;

3. Hybrid-triggered load - heater, boiler, refrigerators etc.- the load which operates according to the daily schedule (depending on consumption), but it can also switch on a little earlier when the price per electricity is economically profitable;

4. Price-responsive load (see Fig.2c) - controllable illumination - the load which changes the consumed power depending on the price per electricity.

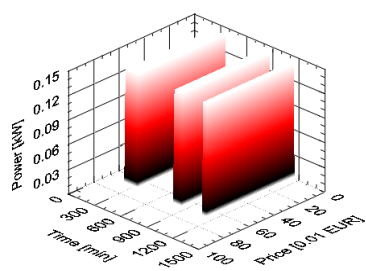

a) Time-triggered load

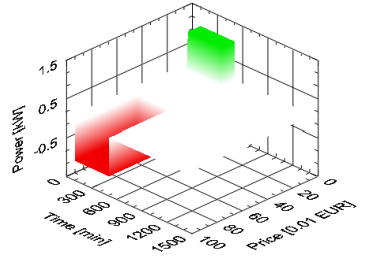

b) Price-triggered storage element

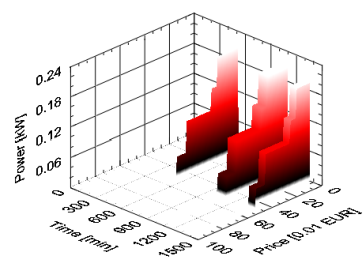

c) Price-responsive load

Fig. 2. Load pattern graphs

The proposed algorithm is realized in the following way. Each load based on the statistics of use and on the changes of electrical price provides an energy management system (EMS) with a schedule of operation (for time-triggered and hybrid-triggered loads), condition of operation (for price-triggered and hybrid-triggered loads): 


$$
\begin{gathered}
\Psi_{\text {load }}^{(1)}(C, t)=\sum_{i}\left(P_{T-\text { trig }}^{i}(C, t)\right)+\sum_{j}\left(P_{C-\text { resp }}^{j}(C, t)\right), \\
\Psi_{\text {source }}(C, t)=\sum_{i}\left(P_{\text {source }}^{i}(C, t)\right) .
\end{gathered}
$$

After having aggregated all data from loads $\left(P_{T-t r i g}^{i}(C, t)\right.$ for time-triggered loads and $P_{C-\text { resp }}^{j}(C, t)$ for price-responsive loads), EMS subtracts the 3D surface of demand $\Psi_{\text {load }}^{(1)}(C, t)$ (to which the power reserve is added $\Psi_{\text {delta }}(C, t)$ ), from the 3D surface of generation $\Psi_{\text {source }}(C, t)$ (see Fig. 3a) to extract the intersection line, the projection of which to Time-Cost axes is used to find the price function $C^{(1)}(t)$ (see Fig. 3b):

$$
\begin{gathered}
\Psi_{\text {source }}(C, t)-\Psi_{\text {load }}^{(1)}(C, t) \geq \Psi_{\text {delta }}(C, t), \\
C^{(1)}(t)=\Psi_{\text {source }}(C, t) \cap\left(\Psi_{\text {load }}^{(1)}(C, t)+\Psi_{\text {delta }}(C, t)\right) .
\end{gathered}
$$

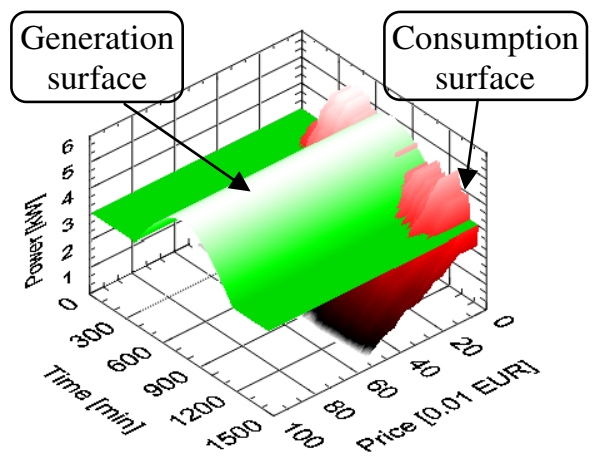

a) Primary energy balancing with time-triggered and price-responsive loads

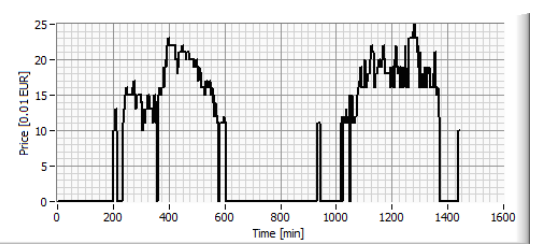

b) Preliminary price function

Fig. 3. Primary equalization of price function

The scheduling of price-triggered loads is also used for the determination of the price function. EMS combines the price-triggered loads in accordance with energy excess, which is calculated using (5) (see Fig.4a):

$$
\begin{gathered}
\Psi_{\text {excess }}(C, t)=\Psi_{\text {source }}(C, t)-\left(\Psi_{\text {load }}^{(1)}(C, t)+\Psi_{\text {delta }}(C, t)\right), \\
\Psi_{\text {excess }}(C, t) \geq \sum_{i} P_{C-\text { trig }}^{i}\left(C^{i},\left.t^{i}\right|_{t_{\text {start }}} ^{t_{\text {end }}}\right),\left\{\begin{array}{l}
C^{i}<C^{i-1} \\
t_{\text {satrt }}^{i} \geq t_{\text {start }}^{i-1} \\
t_{\text {start }}^{i}+t_{\text {min }}^{i} \leq t_{\text {end }}^{i} \leq t_{\text {start }}^{i}+t_{\text {max }}^{i}
\end{array} .\right.
\end{gathered}
$$


As a result, the price function $C^{(1)}(t)$ is changed in accordance with the switching schedule of price-triggered loads, keeping the price at a desirable level, to regulate the amount of switched price-triggered loads and the power balance (see Fig.3b).

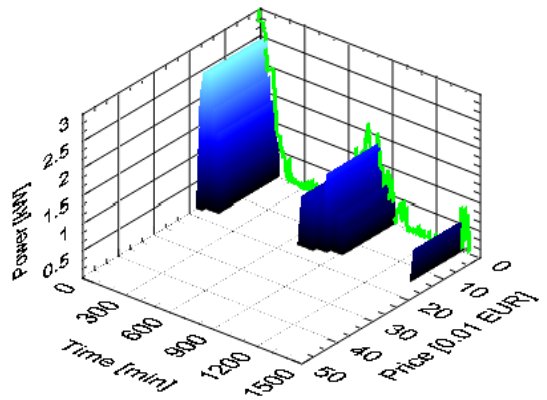

a) Secondary balancing (energy excess is projected to Time-Power axis;3D surface scheduled price-triggered loads)

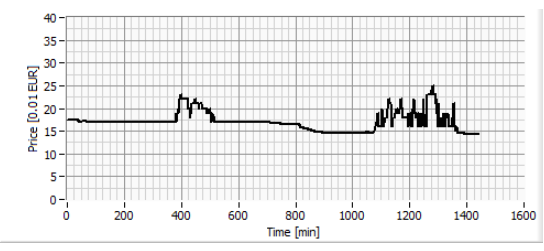

b) Resulting price per electrical energy

Fig. 4. Proposed algorithm of finding the price function

\section{Conclusions and Future Work}

This paper proposes an improved real-time power balancing approach for DC distribution grids. It is based on the possibility of changing the reference parameter of the DC voltage for a bidirectional AC/DC interface converter influencing the DC bus voltage. When the voltage is decreased, it will lead to the change of operation of grid elements controlled by the droop control method, unnecessary loads will be switched off during the period of low DC voltage. Consequently, a larger amount of the energy will be exported to the utility grid. Thus, it is possible to fulfil long-term objectives just by controlling the voltage reference value of a bidirectional interface converter.

The novel approach of long-term energy management is based on pricing technique. It uses the load categorization that defines different conditions of operation for different loads. Each grid element can communicate with the energy management system (EMS), which aggregates time scheduling and condition of operation of all elements and finds the intersection line between the 3D surface of the load and the 3D surface of generation. As a result, a certain price function is defined that can be used to realize predictive energy management of an intelligent distribution grid.

Our future plan is to conduct experiments to test the proposed methods on a small-scale network to acquire real evaluation of long-term and short-term energy management approaches taking into account typical summer, winter and spring day consumption patterns, as well as appropriate forecast generation patterns.

Acknowledgements. This research is co-financed by the European Regional Development Fund within the project "Intellectual Hybrid Uninterruptible Power Systems and Component Development and Research to Improve Energy Efficiency". Project agreement No. 2010/0225/ 2DP/2.1.1.1.0/10/APIA/VIAA/160. 


\section{References}

1. Masson, G., Latour, M., Biancardi, D.: Global market outlook for photovoltaic until 2016, Brussels (2012)

2. Jimeno, J., Anduaga, J.: Architecture of a microgrid energy management system. European Transactions on Electrical Power 21, 1142-1158 (2011)

3. Vermesan, O., Friess, P.: Internet of things strategic research roadmap (2009)

4. Smith, I.G.: Internet of Things 2012 New Horizons, Halifax (2012)

5. Atzori, L., Iera, A., Morabito, G.: The Internet of Things: A survey. Computer Networks 54, 2787-2805 (2010)

6. Bryan, J., Duke, R., Round, S.: Decentralized generator scheduling in a nanogrid using DC bus signaling. In: Power Engineering Society General Meeting, vol. 1, pp. 977-982. IEEE (2004)

7. Cvetkovic, I., Dong, D., Zhang, W., Jiang, L., Boroyevich, D., Lee, F.C., Mattavelli, P.: A Testbed for Experimental Validation of a Low-voltage DC Nanogrid for Buildings. In: 2012 15th International Power Electronics and Motion Control Conference, EPE/PEMC, pp. LS7c.51-LS7c.58 (2012)

8. Piagi, P., Lasseter, R.H.: Autonomous control of microgrids. In: Power Engineering Society General Meeting, p. 8. IEEE (2006)

9. Guerrero, J.M., Vasquez, J.C., Teodorescu, R.: Hierarchical control of droop-controlled AC and DC microgrids - a general approach toward standardization. In: Industrial Electronics Conference, pp. 4341-4346 (2011)

10. Schonberger, J., Round, S., Duke, R.: Autonomous Load Shedding in a Nanogrid using DC Bus Signalling. In: 32nd Annual Conference on IEEE Industrial Electronics, IECON 2006, pp. 5155-5160 (2006)

11. Kanchev, H., Lu, D., Colas, F., Lazarov, V., Francois, B.: Energy Management and Operational Planning of a Microgrid With a PV-Based Active Generator for Smart Grid Applications. IEEE Transactions on Industrial Electronics 58, 4583-4592 (2011)

12. Reddy, Y.J., Kumar, Y.V.P., Kumar, V.S., Raju, K.P.: Distributed ANNs in a Layered Architecture for Energy Management and Maintenance Scheduling of Renewable Energy HPS Microgrids (2012)

13. Logenthiran, T., Srinivasan, D.: Short term generation scheduling of a Microgrid. In: TENCON 2009 - 2009 IEEE Region 10 Conference, pp. 1-6 (2009)

14. Ogimoto, K.: Optimum Operation Scheduling Model of Domestic Electric Appliances for Balancing Power Supply and Demand (2010) 